\title{
SOME APPLICATIONS OF CERTAIN POLYNOMIAL CLASSES ${ }^{1}$
}

I. M. SHEFFER

The term applications will be construed broadly enough as to include properties. It is here proposed, then, to examine some properties and some applications of some classes of polynomial sets.

1. Formal properties of Appell sets. The power series approach to the theory of analytic functions presents us with a very simple polynomial set. ${ }^{2}$ Thus, if $f(x)$ is analytic about $x=a$, we have the expansion

$$
f(x)=\sum_{n=0}^{\infty} c_{n} \frac{(x-a)^{n}}{n !}, \quad c_{n}=f^{(n)}(a),
$$

in terms of the set

$$
\left\{(x-a)^{n} / n !\right\} .
$$

This set has the important property of reproducing itself under the operation of differentiation, in accordance with the rule.

$$
\frac{d}{d x} P_{n}(x)=P_{n-1}(x)
$$

where

$$
P_{n}=(x-a)^{n} / n !
$$

Now this set is not uniquely determined by (2). There are in fact infinitely many sets of polynomials $\left\{P_{n}\right\}$ that satisfy (2). These bear the name of $A$ ppell sets,$^{3}$ after the man who in $1880[1]^{4}$ first made a study of them. Appell sets will be the first class to be considered here.

There are many conditions that are equivalent to the defining relation (2) for Appell sets. Among the simplest are the following two:

\footnotetext{
${ }^{1}$ An address delivered before the meeting of the Society in Washington, D.C., May 3, 1941, by invitation of the Program Committee.

${ }^{2}$ By a set $\left\{P_{n}\right\}$ we understand an infinite sequence $P_{0}, P_{1}, \cdots$, with $P_{n}$ of degree $n$.

${ }^{3}$ Strictly speaking, Appell's definition is

$$
\frac{d}{d x} P_{n}(x)=n P_{n-1}(x),
$$

but (2) is preferable for our purpose. If $\left\{P_{n}\right\}$ satisfies (2), then $\left\{n ! P_{n}\right\}$ satisfies (2'), and conversely. A bibliography on Appell polynomials is given in Davis [5].

${ }^{4}$ Numbers in brackets refer to the bibliography placed at the end.
} 
(i) A set $\left\{P_{n}\right\}$ is an $A$ ppell set if and only if constants $\left\{a_{n}\right\}$ exist $\left(a_{0} \neq 0\right)$ such that

$$
P_{n}(x)=a_{0} \frac{x^{n}}{n !}+a_{1} \frac{x^{n-1}}{(n-1) !}+\cdots+a_{n} \frac{x^{0}}{0 !}, \quad n=0,1, \cdots .
$$

(This is the explicit representation of an Appell set.) (ii) In order that $\left\{P_{n}\right\}$ be an Appell set it is necessary and sufficient that a formal power series

$$
A(t)=\sum_{0}^{\infty} a_{n} t^{n}
$$

exist so that (formally)

$$
A(t) e^{t x}=\sum_{0}^{\infty} P_{n}(x) t^{n}
$$

$A(t)$ will be called the generating function for $\left\{P_{n}\right\}$.

It is natural to inquire, regarding a set of polynomials, if it has properties analogous to those of the classical sets (Legendre, Hermite, and so on). For example, does it satisfy a linear differential equation of the form

$$
L_{0} y+L_{1} y^{\prime}+L_{2} y^{\prime \prime}=\lambda y
$$

where $L_{i}$ is a polynomial of degree not exceeding $i$, and $\lambda$ is a parameter which takes on a value $\lambda_{n}$ for the $n$th polynomial of the set? Or an equation of the same type but of higher order? Such equations contain too few constants to serve for all Appell sets, although we shall see that there is a subclass of such sets that satisfy equations of this type. If, however, we are willing to permit the order to become infinite:

$$
\sum_{r=0}^{\infty} L_{r}(x) y^{(r)}(x)=\lambda y(x)
$$

where $L_{r}$ is of degree not exceeding $r$, then there is ample freedom not only for Appell sets but for all sets. That is, (6) is a universal equation for all polynomial sets in that given an arbitrary set $\left\{P_{n}\right\}$, it is possible to choose $\left\{L_{r}\right\},\left\{\lambda_{r}\right\}$, and indeed in infinitely many ways, so that $\left\{P_{n}\right\}$ satisfies (6) $\left(y=P_{n}\right.$ for $\left.\lambda=\lambda_{n}\right)$.

The advantages of a universal or, as we may say, canonical equation are evident. But this very generality may sometimes be a defect in studying particular sets, unless it is possible to characterize the coefficients $\left\{L_{r}\right\}$ corresponding to such sets. Fortunately a charac- 
terization can be achieved in the case of Appell sets. The simplest (but not the only ${ }^{5}$ ) canonical equation of form (6) for Appell sets ${ }^{6}$ is [11]

$$
\left(b_{0}+x\right) P_{n}^{\prime}+b_{1} P_{n}^{\prime \prime}+b_{2} P_{n}^{\prime \prime \prime}+\cdots=n P_{n}, \quad n=0,1, \cdots .
$$

That is, $L_{0}=0, L_{1}=b_{0}+x, L_{k}=b_{k-1}, k \geqq 2 ; \lambda_{n}=n$. Moreover, if $\left\{P_{n}\right\}$ has the generating function $A(t)$, then $\left\{b_{n}\right\}$ is defined by

$$
\sum_{0}^{\infty} b_{n} t^{n} \equiv B(t)=A^{\prime}(t) / A(t) .
$$

It follows from (7) that $\left\{P_{n}\right\}$ is an Appell set if and only if a sequence $\left\{b_{n}\right\}$ exists so that

(9) $n P_{n}(x)=\left(b_{0}+x\right) P_{n-1}+b_{1} P_{n-2}+\cdots+b_{n-1} P_{0}, n=1,2, \cdots$.

This is of interest because it gives the set $\left\{P_{n}\right\}$ by recurrence. It has been used by Webster [17] to give a simple proof that the Hermite polynomial set is the only Tchebycheff (orthogonal) set that is at the same time an Appell set. Here then is an Appell set that satisfies a second order equation of form (6). In general we have the theorem: An Appell set $\left\{P_{n}\right\}$, with generating function $A(t)$, satisfies a finite order equation of type (6) if and only if $A(t)$ has the form

$$
A(t)=e^{Q(t)}, \quad Q(t)=\text { polynomial; }
$$

and the minimum order of all such equations for $\left\{P_{n}\right\}$ is the degree of $Q(t)$ [11]. As examples, the set $\left\{(x-a)^{n} / n !\right\}$ and the Hermite set have for $A(t)$ the respective functions $e^{-a t}, e^{-t^{2} / 4}$; and these sets satisfy equations of the first and second order respectively.

2. Relation of Appell sets to functional equations. In 1888 Pincherle made a study of the following linear difference equation with constant coefficients :

$$
L[y(x)] \equiv \sum_{j=1}^{k} a_{j} y\left(x+\omega_{j}\right)=F(x) .
$$

His memoir, in a French translation, was reprinted in 1926 in Acta Mathematica [10]. In the course of his investigation he pointed out the value of Appell sets. Thus for (11), two Appell sets preëminently suggest themselves, namely $\left\{P_{n}\right\},\left\{Q_{n}\right\}$, defined respectively by

5 There are infinitely many.

${ }^{6}$ Conversely, if $\left\{P_{n}\right\}$ satisfies (7), then $\left\{c_{n} P_{n}\right\}$ will be an Appell set for proper choice of the constants $\left\{c_{n}\right\}$.

${ }^{7}$ The one exception is when $Q(t) \equiv$ constant. 


$$
L\left[x^{n} / n !\right]=P_{n}(x), \quad L\left[Q_{n}(x)\right]=x^{n} / n ! .
$$

For as Pincherle observed, if $F(x)$ has the expansion

$$
F(x)=\sum_{0}^{\infty} c_{n} P_{n}(x)
$$

then a formal solution of (11) is given by

$$
y(x)=\sum_{0}^{\infty} c_{n} x^{n} / n !
$$

and if $F(x)$ has the expansion (1) (for $a=0$ ), then

$$
y(x)=\sum_{0}^{\infty} c_{n} Q_{n}(x)
$$

formally satisfies (11). For the case that $F(x)$ is an entire function of finite exponential type, ${ }^{8}$ Pincherle showed that there is a solution of (11) of the same kind. He also found a solution when $F(x)$ is analytic at infinity. His method of proof however did not involve polynomial sets.

In discussing the equation

$$
L[y(x)] \equiv y(x+1)-y(x)=F(x),
$$

a particular case of (11), A. Hurwitz [7] used the set $\left\{Q_{n}\right\}$, which for (16) is the set of Bernoulli polynomials. Since series in these polynomials serve to define only a limited class of entire functions, whereas he was interested in the case where $F(x)$ is an arbitrary entire function, Hurwitz modified the polynomials by adding to them linear combinations of properly chosen exponential functions. The device used has been termed the method of expanding contours. For from the generating relation

$$
\frac{t e^{t x}}{e^{t}-1}=\sum_{0}^{\infty} Q_{n}(x) t^{n}
$$

for the Bernoulli set, valid for $|t|<2 \pi$, follows the formula

$$
Q_{n}(x)=\frac{1}{2 \pi i} \int_{C} \frac{e^{t x} d t}{\left(e^{t}-1\right) t^{n}}
$$

$C$ being a contour around $t=0$ and lying in $|t|<2 \pi$. If now $C$ is expanded so as to include a certain number of poles of the integrand, the

${ }^{8}$ A definition will be given later. 
new contour, call it $C_{n}$, will define for each $n=0,1, \cdots$ a function

$$
Q_{n}^{*}(x)=\frac{1}{2 \pi i} \int_{C_{n}} \frac{e^{t x} d t}{\left(e^{t}-1\right) t^{n}},
$$

which is $Q_{n}(x)$ augmented by certain exponentials (the residues at the poles lying within $C_{n}$ ). Hurwitz proved that to every entire function $F(x)$ given by (1) $(a=0)$ there is an entire function solution of (16) given by (15) with $Q_{n}$ replaced by $Q_{n}^{*}$.

Carmichael [4] extended the method and the conclusion to equation (11). The method of expanding contours can be shown to apply to (11) and (16) even when $F(x)$ is not an entire function. All that is required is that $F(x)$ be analytic about some point in a circle of radius exceeding a number $r$, whose (fixed) value is determined by the equation and the method. That is, one obtains what may be called a semilocal solution rather than a completely local one.

This restrictive condition on $F(x)$ can be, and is, lifted by emphasizing the set $\left\{P_{n}\right\}$ rather than $\left\{Q_{n}\right\}[12],[13]$. For equation (11), $P_{n}(x)$ is given by

$$
P_{n}(x)=\frac{1}{n !}\left[a_{1}\left(x+\omega_{1}\right)^{n}+\cdots+a_{k}\left(x+\omega_{k}\right)^{n}\right] .
$$

Let the points $-\omega_{j}, j=1, \cdots, k$, be plotted. For each $x$ let

$$
\rho_{j}(x)=\left|x+\omega_{j}\right|, \quad \rho(x)=\max \left\{\rho_{1}(x), \cdots, \rho_{k}(x)\right\} .
$$

As $x$ varies, there is a unique point $x^{*}$ at which $\rho(x)$ attains its minimum value $\rho=\rho^{*}$. This means that the circle of radius $\rho^{*}$ and center $x^{*}$ will cover the set $\left\{-\omega_{j}\right\}$, and that no other circle of equal or smaller radius will have this property.

Now consider (20). It is undesirable, from the point of view of convergence, to consider those values of $x$ for which the maximum $\rho(x)$ is attained by two or more of the $\rho_{j}(x)$ 's. The set of such points $x$, which we shall term the critical set, is to be excepted. The following results can be proved: If the series $\sum n ! c_{n} P_{n}(x)$ converges at $x=x_{0}$ (not in the critical set), then it converges absolutely in the circular polygon

$$
\rho_{j}(x)=\left|x+\omega_{j}\right|<\rho\left(x_{0}\right), \quad j=1, \cdots, k,
$$

and uniformly in every closed region therein; and a necessary and suffcient condition that the above series converge for at least one $x$ not in the critical set is that lim sup $\left|c_{n}\right|^{1 / n}</ 1 \rho^{*}$.

When such a series converges, and thus converges uniformly in some region, the function defined by the series is of course analytic. 
The converse is also true: If and only if a function $F(x)$ is analytic at $x=x^{*}$ can it be expressed as a convergent $P_{n}$-series. The manner of proof is roundabout. Set $F(x)=1 /(x-\alpha)$ in (11), $\alpha$ being a parameter. The resulting equation

$$
L[W(x ; \alpha)]=\frac{1}{x-\alpha}
$$

is shown to have two (or three, according to the case) meromorphic solutions, from which follow two (or three) $P_{n}$-expansions for $1 /(x-\alpha)$, valid in some neighborhood of $x=x^{*}$, for $\alpha$ on two (or three) arcs that form a closed contour around $x^{*}$. Application of the Cauchy integral formula then shows that $F(x)$, analytic at $x^{*}$, also has a convergent $P_{n}$-series. It follows, also, that if $F(x)$ is analytic about $x^{*}$ there is an analytic solution of (11) in the neighborhood of $x^{*}$; and a translation of the variable $x$ permits the point $x^{*}$ to be replaced by any other point of the plane.

It should be added that Appell expansions are usually not unique; that is, that the function zero possesses at least one $P_{n}$-expansion in which the coefficients are not all zero. To see this we turn back to (5). If $t_{0} \neq 0$ is a zero of the generating function $A(t)$, then

$$
0=\sum_{0}^{\infty} t_{0}^{n} P(x)
$$

is an expansion of zero.

We now consider the more general functional equation

$$
A[y(x)] \equiv \sum_{k=0}^{\infty} a_{k} y^{(k)}(x)=F(x),
$$

which is a linear differential equation of infinite order, with constant coefficients. Equation (11) is subsumed under (24). For if we call

$$
A(t)=\sum_{0}^{\infty} a_{k} t^{k}
$$

the generating function for operator $A[y]$, then (11) is that case of (24) for which

$$
A(t)=\sum_{j=1}^{k} a_{j} e^{\omega_{j} t} .
$$

The Appell set $\left\{P_{n}\right\}$ defined by (12) likewise has as its generating function the $A(t)$ of (25). Now the expansion problem will differ with the character of $A(t)$. In general, the larger the class of functions that 
we wish to expand in a $\left\{P_{n}\right\}$-series, the greater must be the restriction on $A(t)$. A fruitful assumption, and one that we now make, is that $A(t)$ is of finite exponential type. ${ }^{9}$ That is, the coefficients of (25) satisfy the inequality

$$
\limsup _{n \rightarrow \infty}\left|n ! a_{n}\right|^{1 / n}<\infty .
$$

If this superior limit has the value $\sigma$, then $A(t)$ is said to be of $e x-$ ponential type $\sigma$ (exp. type $\sigma)$.

From (6) we see that $A(t) e^{t x}$ is, in the variable $t$, of exp. type not exceeding $\sigma+|x|$, so that lim sup $\left|n ! P_{n}(x)\right|^{1 / n} \leqq \sigma+|x|$. This is, however, too crude an estimate. To better it, we observe that if a function $C(t)=\sum_{0}^{\infty} c_{n} t^{n}$ is of exp. type $\sigma$, then it is the associated entire function $(A E F)$ relative to the function $C_{1}(t)=\sum_{0}^{\infty} n ! c_{n} t^{n}$; and this latter function has $1 / \sigma$ as its radius of convergence. Conversely, if by studying $C_{1}(t)$ one can locate the singularity nearest the origin, that will serve to determine the type of $C(t)$.

Applying this principle to (5), it is found [14] on setting

$$
A(t)=\operatorname{AEF}\left\{A_{1}(t)\right\}, \quad A^{*}(t)=t A_{1}(t),
$$

that

$$
A^{*}\left(\frac{1}{t-x}\right)=\sum_{0}^{\infty} \frac{n ! P_{n}(x)}{t^{n+1}},
$$

$|t|$ sufficiently large. Let $G=\{\alpha\}$ be the set of all the singularities of $A^{*}(t)$ in the whole plane, and define the continuous function $D(x)$ by

$$
D(x)=\max _{G}|x+1 / \alpha| \text {. }
$$

It can then be established that

$$
\lim \sup \left|n ! P_{n}(x)\right|^{1 / n}=D(x) .
$$

Let us call the curves $D(x)=c$ the level curves for the set $\left\{P_{n}\right\}$. A point set discussion reveals the following information: There is a unique point $x^{*}$ where $D(x)$ has its minimum value $D_{m}$. For every $c>D_{m}$, the level curve is a simple closed convex curve, containing in its interior the level curves $D(x)=c^{\prime}$ for every $c^{\prime}$ in $D_{m} \leqq c^{\prime}<c$. In the case that the points $\{\alpha\}$ are finite in number, the level curves consist of circular arcs. This is the situation relative to equation (11), where

${ }^{9}$ See Muggli [9], where other cases are also taken up. The solution of (24) is there carried out by the method of expanding contours, and for the case where $A(t)$ is of finite exponential type, $F(x)$ is assumed to be an entire function. 
the region of convergence of a $P_{n}$-expansion was found to be a circular polygon.

With respect to the present general case we find that: If lim $\sup \left|c_{n}\right|^{1 / n}$ $=\rho<1 / D_{m}$, then series $\sum n ! c_{n} P_{n}(x)$ converges absolutely interior to the level curve $D(x)=1 / \rho$, and converges uniformly in every closed region therein, thus representing an analytic function there.

At this point we are forced to abandon the method that proved so useful in dealing with (11), for we have not sufficient knowledge of solutions of the equation corresponding to (22) to follow the earlier plan. Turning to the polynomial set $\left\{Q_{n}\right\}$ of (12), and modifying it by the method of expanding contours, it is possible, first to solve equation (24) semi-locally, and then to obtain a $P_{n}$-expansion theorem: If $F(x)$ is analytic about a point $x=x_{0}$ in a circle of radius exceeding $\lambda$, where $\lambda$ is determined by the operator $A$, then equation (24) has an analytic solution $y(x)$ in some neighborhood of $x_{0}$; and if $F(x)$ is analytic about $x=0$ in a circle of radius exceeding $\lambda^{*}$ (again determined by operator $A$ ), then $F(x)$ has a convergent Appell expansion

$$
F(x)=\sum_{0}^{\infty} n ! c_{n} P_{n}(x) \text {. }
$$

The numbers $\lambda, \lambda^{*}$ are not sharply defined. Burdette [3] has obtained sharp values on making the additional hypothesis that $A(t)$ is bounded on a certain infinite sequence of expanding contours.

3. Sets of type zero. Let us turn back to equation (16). Relative to it one can consider the set of Newton polynomials

$$
N_{n}(x)=\frac{x(x-1) \cdots(x-n+1)}{n !} ;
$$

for this set, which is not an Appell set, has the fundamental property of reproducing itself under application of the difference operator:

$$
\Delta N_{n}(x)=N_{n-1}(x),
$$

so that if $F(x)$ has the expansion

$$
F(x)=\sum_{0}^{\infty} c_{n} N_{n}(x),
$$

then a formal solution of (16) is given by

$$
y(x)=\sum_{0}^{\infty} c_{n} N_{n+1}(x) .
$$


We may say that $\left\{N_{n}\right\}$ is a set of difference polynomials, as is every other set (and there are infinitely many such) that has the property (34). Such sets may be examined as were Appell sets, but at this point there is suggested a general problem: Can there be assigned to an arbitrary set $\left\{P_{n}\right\}$ an operator $J$ that will reproduce $\left\{P_{n}\right\}$ in the sense that

$$
J\left[P_{n}(x)\right]=P_{n-1}(x)
$$

for all $n$ ?

The answer is in the affirmative, and $J$ can be expressed in the form

$$
J[y] \equiv \sum_{n=1}^{\infty} L_{n-1}(x) y^{(n)}(x)
$$

where $L_{k}(x)$ is a polynomial of degree not exceeding $k$. This too is a universal (or canonical) operator, since to every set corresponds a determined $J$. For Appell sets and for difference sets, the $L_{n}$ 's are constants. This suggests examination of all sets with constant $L_{n}$ 's. We shall say that $a$ set $\left\{P_{n}\right\}$ is of zero type [15] if in its associated operator $J$ (as given by (38)) the polynomials $L_{n}(x)$ are all constants.

For zero type sets, then, (38) can be written

$$
J[y(x)] \equiv \sum_{n=1}^{\infty} c_{n} y^{(n)}(x), \quad c_{1} \neq 0 .
$$

Let the formal power series

$$
J(t) \equiv \sum_{1}^{\infty} c_{n} t^{n}
$$

be called the generating function for (39), and let $H(t)$ be the inverse power series to $J(t)$, so that (formally)

$$
J(H(t))=H(J(t))=t .
$$

Zero type sets can then be characterized as follows: $\left\{P_{n}\right\}$ is a set of type zero if and only if a formal power series $A(t)=\sum_{0}^{\infty} a_{n} t^{n}$ exists so that

$$
A(t) e^{x H(t)}=\sum_{0}^{\infty} P_{n}(x) t^{n}
$$

Observe that the Laguerre polynomials $\left\{L_{n}(x)\right\}$ are of zero type, since

$$
\frac{1}{1-t} \exp \left(\frac{-x t}{1-t}\right)=\sum_{0}^{\infty} L_{n}(x) t^{n} \text {. }
$$


Various other characterizations of zero type sets can be found. One of the simplest is that constants $\left\{q_{k 0}\right\},\left\{q_{k 1}\right\}$ exist so that the recurrence relation

$$
\sum_{k=1}^{\infty}\left(q_{k 0}+x q_{k 1}\right) P_{n-k}(x)=n P_{n}(x)
$$

holds for $n=1,2, \ldots$. The relation between (42) and (44) is expressed by the series

$$
\frac{A^{\prime}(t)}{A(t)}=\sum_{0}^{\infty} q_{n+1,0} t^{n}, \quad H^{\prime}(t)=\sum_{0}^{\infty} q_{n+1,1} t^{n} .
$$

Having learned that among Appell sets is a Tchebycheff set (the Hermite polynomials) one naturally asks if there are other orthogonal sets in the extended class of zero type sets. This problem was solved by Meixner [8], by use of the Laplace transformation. He took (42) as his definition of the polynomials that he was investigating. Following Meixner, Geronimus obtained some interesting properties of zero type sets. A second resolution of the Meixner problem can be made by the combined used of (44) and the characterizing recurrence relation

$$
Q_{n}(x)=\left(x+\lambda_{n}\right) Q_{n-1}(x)+\mu_{n} Q_{n-2}(x), \quad n=1,2, \cdots,
$$

for orthogonal polynomials. For (46) to represent a set of type zero it is necessary and sufficient that

$$
\lambda_{n}=a+b n, \quad \mu_{n}=(n-1)(c+d n),
$$

with $c+d n \neq 0$ for $n>1$. From this will follow the explicit determination of the required sets, as (otherwise) obtained by Meixner.

Consider an operator $J$ of form (39). The equation (24) can be equally well expressed in terms of $J$ :

$$
A[y(x)] \equiv \sum_{k=0}^{\infty} a_{k}^{*} J^{k}[y(x)]=F(x),
$$

where $J^{k}[y] \equiv J\left[J^{k-1}[y]\right], J^{0}[y] \equiv y$. As such, we see that polynomial sets of zero type suggest themselves as a means of studying equation (24). This study, however, awaits more knowledge of the expansion properties of such sets.

4. On equations with non-constant coefficients. The functional equations already considered have been of the constant coefficient type, and it may be asked if zero type sets can be applied to more general equations. Such application has been made to the linear differ- 
ential equation of finite order, and this points the way to other equations (where, however, the results are as yet only formal).

Consider the differential equation

$$
L[y(x)] \equiv y^{(k)}+\rho_{1}(x) y^{(k-1)}+\cdots+\rho_{k}(x) y=F(x) .
$$

We seek [16] an operator $M[y]$ that is inverse to $L[y]$ in accordance with the relations

$$
M L[y] \equiv y ; \quad L M[y] \equiv y .
$$

The assumed form of $M[y]$ is

$$
M[y] \equiv \sum_{n=-\infty}^{+\infty} M_{n}(x) y^{(n)}(x)
$$

where

$$
y^{(-n)}(x)=\int_{x_{0}(n-\text { fold })}^{x} \ldots \int_{x_{0}}^{x} y(t) d t, \quad n=1,2, \cdots .
$$

It is found that (50) can be satisfied by choosing $M_{n}(x)=0, n>-k$; $M_{-k}(x)=1$; and the other $M$ 's by recurrence:

$$
\begin{array}{rlrl}
M_{-m}(x)= & C_{m, m-1}(x) M_{-(m-1)}(x)+C_{m, m-2}(x) M_{-(m-2)}(x)+ & \cdots \\
& +C_{m, k}(x) M_{-k}(x), \quad m>k .
\end{array}
$$

(The functions $C_{i j}(x)$ are determined by the coefficients $\rho_{n}(x)$ and their derivatives.) On setting

$$
H(x, t)=M_{-k}(x) \frac{(x-t)^{k-1}}{(k-1) !}+M_{-(k+1)}(x) \frac{(x-t)^{k}}{k !}+\cdots,
$$

a solution of (49) is given by

$$
y(x)=\int_{x_{0}}^{x} H(x, t) F(t) d t .
$$

$H(x, t)$ itself satisfies (in the variable $x$ ) the homogeneous equation

$$
L[u]=0 \text {, }
$$

as do also $\partial H / \partial t, \partial^{2} H / \partial t^{2}, \cdots, \partial^{k-1} H / \partial t^{k-1}$; and these $k$ functions form an independent set of solutions.

The series (54) has an interesting domain of convergence, but we must forego a discussion of this [16]. What is of importance for our present aim is the observation that the Appell polynomial set $\left\{(x-t)^{n} / n !\right\}$ is present (at least, beginning with $n=k-1$ ) in this 
series. Any other Appell set would (formally at least) lead to the same result, in the following sense: If $\left\{P_{n}(x)\right\}$ is an Appell, set then the formal series

$$
K(x, t)=M_{-k}(x) P_{k-1}(x-t)+M_{-(k+1)}(x) P_{k}(x-t)+\cdots
$$

satisfies (56). The convergence problem for (57) has not been examined, although relation (31) is certainly applicable.

With (49) as a guide, we turn to the corresponding difference equation

$$
L[u] \equiv \Delta^{k} u(x)+q_{1}(x) \Delta^{k-1} u(x)+\cdots+q_{k}(x) u(x)=F(x) .
$$

Here we look for an inverse operator $M(L M[u] \equiv M L[u] \equiv u)$ in the form

$$
M[u] \equiv \sum_{n=-\infty}^{+\infty} M_{n}(x) \Delta^{n} u(x),
$$

where for negative $n$ the "sum" is meant; that is, $\Delta^{n}\left[\Delta^{-n}[u]\right]=u$. We can again choose $M_{n}(x)=0$ for $n>-k ; M_{-k}(x)=1$; after which $M_{-(k+1)}(x), M_{-(k+2)}(x), \cdots$ are determined by recurrence, in analogy with (53). And like (54) (or (57)) we set up the (formal) series

$$
K(x, t)=M_{-k}(x) P_{k-1}(x-t)+M_{-(k+1)}(x) P_{k}(x-t)+\cdots
$$

where $\left\{P_{n}\right\}$ is any difference set $\left(\Delta P_{n}=P_{n-1}\right)$.

Convergence has not yet been examined. Formally, however, (60) satisfies in the variable $x$ the homogeneous equation $L[u]=0$, as do the functions $\Delta K, \Delta^{2} K, \cdots$, where the differences are taken with respect to the variable $t$.

5. Remarks on null-functions. We shall close with some remarks on the problem of null-functions (as we may call them). Let an infinite sequence of functionals $\left\{\mathcal{X}_{n}\right\}$ be given. We assume them to be of the form

(61) $\mathcal{X}_{n}[f(x)]=c_{n 0} f(0)+c_{n 1} f^{\prime}(0)+c_{n 2} f^{\prime \prime}(0)+\cdots, n=0,1, \cdots$, supposed applicable to a given class of analytic functions. By a nullfunction we shall understand a function $f(x)$, not identically zero, for which

$$
\mathcal{X}_{n}[f(x)]=0, \quad n=0,1, \cdots .
$$

The determination of null-functions is linked to the problem of finding the radius of completeness of the sequence of functions $\left\{M_{n}(t)\right\}$, where 


$$
M_{n}(t)=c_{n 0}+c_{n 1} t+c_{n 2} t^{2}+\cdots ;
$$

that is, the largest number $r$ such that every function $f(t)$, analytic in $|t|<r$, has an expansion

$$
f(t)=\sum_{0}^{\infty} f_{n} M_{n}(t)
$$

convergent in $|t|<r$ and uniformly convergent in every closed region therein. ${ }^{10}$

An interesting subclass of (61) is that in which $\mathcal{X}_{n}$ has the form

$$
\mathcal{X}_{n}[f]=c_{n n} f^{(n)}(0)+c_{n, n+1} f^{(n+1)}(0)+\cdots, \quad c_{n n} \neq 0 .
$$

An example of this, namely when

$$
\mathcal{X}_{n}[f]=f^{(n)}\left(a_{n}\right), \quad\left|a_{n}\right| \leqq 1, \quad n=0,1, \cdots,
$$

leads to the Takenaka problem [18] of determining the largest number $\lambda$ for which it is true that no entire function of exp. type less than $\lambda$ can be a null-function. It is known that $\lambda \geqq \log 2$, and it is believed that $\lambda=\pi / 4$. The example

$$
f(x)=\cos \frac{\pi x}{4}-\sin \frac{\pi x}{4}, \quad a_{n}=(-1)^{n},
$$

shows that $\pi / 4$ cannot be exceeded.

Corresponding to the above sequence $a_{n}=(-1)^{n}$ (and it may very well be true for every sequence for which $\left\{a_{n}\right\}$ is bounded), the following is true: Given any $\sigma>0$, there is only a finite number of linearly independent null-functions of exp. type not exceeding $\sigma$. (As $\sigma \rightarrow \infty$, however, the number of independent null-functions is unbounded.) If, however, the sequence $\left\{a_{n}\right\}$ is not required to be bounded, and in other cases of the functionals $\left\{\mathcal{X}_{n}\right\}$, there may be a continuous array of null-functions. This fact we shall illustrate by borrowing from the theory of sets of type zero. Consider, in fact, the set $\left\{P_{n}\right\}$ for which

$$
e^{x H(t)}=\sum_{0}^{\infty} P_{n}(x) t^{n}
$$

or, letting $J(t)$ be the inverse of $H(t)$,

$$
e^{x t}=\sum_{0}^{\infty} P_{n}(x)[J(t)]^{n} .
$$

${ }^{10}$ For work in this direction, consult Whittaker [18], Gontcharoff [6] and Boas [2]. These works include further references. 
Take $[J(t)]^{n}$ as the function $M_{n}(t)$ of (63), which thus determines the functionals $\mathscr{H}_{n}[f]$.

A simple example is given by $H(t)=t=J(t)$, for then $\mathscr{X}_{n}[f]=f^{(n)}(0)$, so that there is no null-function that is analytic at the origin. Now take $H(t)=\log (t+1), J(t)=e^{t}-1$. One verifies that the function $f(x)=e^{\alpha x}\left(1-e^{2 k \pi i x}\right)$ is a null-function for every integer $k$ and for every value of $\alpha$. That is, there is a continuous spectrum of null-functions. This property is shared by functionals $\left\{\mathcal{X}_{n}\right\}$ corresponding to other choices of $H(t)$.

\section{BIBLIOGRAPHY}

1. P. Appell, Sur une classe de polynômes, Annales Scientifiques de l'Ecole Normale Supérieure, (2), vol. 9 (1880), pp. 119-144.

2. R. P. Boas, Jr., Expansions of analytic functions, Transactions of this Society, vol. 48 (1940), pp. 467-487.

3. A. C. Burdette, On a local solution of a differential equation of infinite order, American Journal of Mathematics, vol. 63 (1941), pp. 291-294.

4. R. D. Carmichael, Systems of linear difference equations $\cdots$, Transactions of this Society, vol. 35 (1933), pp. 1-28.

5. H. T. Davis, The Theory of Linear Operators, The Principia Press, Bloomington, Indiana, 1936.

6. W. Gontcharoff, Détermination des Fonctions Entières par Interpolation, Actualités Scientifiques et Industrielles, no. 465, Paris, 1937.

7. A. Hurwitz, Sur l'intégrale finie d'une fonction entière, Acta Mathematica, vol. 20 (1896-1897), pp. 285-312.

8. J. Meixner, Orthogonale Polynomsysteme mit einer besonderen Gestalt der erzeugenden Funktion, Journal of the London Mathematical Society, vol. 9 (1934), pp. 6-13.

9. H. Muggli, Differentialgleichungen unendlich hoher Ordnung mit konstanten Koeffizienten, Commentarii Mathematici Helvetici, vol. 11 (1938-1939), pp. 151-179.

10. S. Pincherle, Sur la résolution..., Acta Mathematica, vol. 48 (1926), pp. 279-304.

11. I. M. Sheffer, A differential equation for Appell polynomials, this Bulletin, vol. 41 (1935), pp. 914-923.

12. - A local solution of the difference equation $\Delta y(x)=F(x)$ and of related equations, Transactions of this Society, vol. 39 (1936), pp. 345-379.

13. - A correction, Transactions of this Society, vol. 41 (1937), pp. 153-159.

14. - Concerning Appell sets and associated linear functional equations, Duke Mathematical Journal, vol. 3 (1937), pp. 593-609.

15. - - Some properties of polynomial sets of type zero, Duke Mathematical Journal, vol. 5 (1939), pp. 590-622.

16. —_, An aspect of the theory of linear differential equations, Tôhoku Mathematical Journal, vol. 39 (1934), pp. 299-315.

17. M. S. Webster, Orthogonal polynomials with orthogonal derivatives, this Bulletin, vol. 44 (1938), pp. 880-888. 1935.

18. J. M. Whittaker, Interpolatory Function Theory, Cambridge Tracts, no. 33.

\section{Pennsylvania State College}

